\title{
HILBERT-SCHAUDER FRAME OPERATORS
}

\begin{abstract}
RUI LIU
Abstract. We introduce a new concept of frame operators for Banach spaces we call a HilbertSchauder frame operator. This is a hybird between standard frame theory for Hilbert spaces and Schauder frame theory for Banach spaces. Most of our results involve basic structure properties of the Hilbert-Schauder frame operator. Examples of Hilbert-Schauder frames include standard Hilbert frames and classical bases of $\ell_{p}$ and $L^{p}$-spaces with $1<p \leqslant 2$. Finally, we give a new isomorphic characterization of Hilbert spaces.
\end{abstract}

Mathematics subject classification (2010): Primary 46B15, 46B28, 46B45, 47B38; Secondary 47A20. Keywords and phrases: Hilbert spaces, Banach spaces, Hilbert-Schauder frames, Hilbert-Schauder frame operators.

\section{REFERENCES}

[1] F. Albiac And N. J. Kalton, Topics in Banach space theory, Springer, 2006.

[2] D. AlsPaCh AND E. Odell, $L_{p}$ spaces. Handbook of the geometry of Banach spaces, Vol. I, 123159, edited by W. B. Johnson and J. Lindenstrauss, North-Holland Publishing Co., Amsterdam, 2001.

[3] K. BeAnland, D. Freeman AND R. Liu, Upper and lower estimates for Schauder frames and atomic decompositions, preprint, arXiv:1202.2492v1.

[4] D. CARANDo And S. Lass Alle, Duality reflexivity and atomic decompositions in Banach spaces, Studia Math. 191 (2009), 67-80.

[5] D. CARAndo, S. LASS ALle AND P. SchmidBeRG, The reconstruction formula for Banach frames and duality, J. Approx. Theory 163 (2011), 640-651.

[6] P. G. CASAZZA, The art of frame theory, Taiwanese J. Math. 4, 2 (2000), 129-201.

[7] P. G. Casazza, S. J. Dilworth, E. Odell, Th. Schlumprecht and A. Zsak, Coefficient Quantization for Frames in Banach Spaces, J. Math. Anal. Appl. 348 (2008), 66-86.

[8] P. G. Casazza, D. Han AND D. R. Larson, Frames for Banach spaces, Contemp. Math. 247 (1999), 149-182.

[9] O. Christensen, An introduction to frames and Riesz bases, Birkhä user, 2003.

[10] I. Daubechies, A. Grossmann And Y. Meyer, Painless nonorthogonal expansions, J. Math. Physics 27 (1986), 1271-1283.

[11] R. J. Duffin And A. C. Schaeffer, A class of nonharmonic Fourier series, Tran. Amer. Math. Soc. 72 (1952), 341-366.

[12] M. Fabian, P. Habala, P. Hájek, V. M. Santalucía, J. Pelant, V. Zizler, Functional analysis and infinite-dimensional geometry, CMS books in mathematics, Springer, 2001.

[13] D. Freeman, D. Poore, A. R. Wei, And M. Wyse, Moving Parseval frames for vector bundles, preprint.

[14] D. GABOR, Theory of communications, Jour. Inst. Elec. Eng. 93 (1946), 429-457.

[15] K. GRöCHENIG, Describing functions: atomic decompositions versus frames, Monatsh. Math. 112, 1 (1991), 1-42.

[16] D. Han And D. R. LaRson, Frames bases and group representations, Memoirs Amer. Math. Soc. 147, 697 (2000), $\mathrm{x}+94 \mathrm{pp}$.

[17] D. Han, D. R. LaRson, B. LiU And R. Liu, Operator-Valued Measures, Dilations and the Theory of Frames, preprint, arXiv:1110.5833v1.

[18] J. Holub, Pre-frame operators, Besselian frame and near-Riesz bases in Hilbert spaces, Proc. Amer. Math. Soc. 122, 3 (1994), 779-785. 
[19] R. C. JAMES, Bases and reflexivity of Banach spaces, Ann. of Math. 52 (1950), 518—527.

[20] J. Lindenstrauss AND L. TZAFrIRI, Classical Banach spaces I and II, Springer, 1996.

[21] R. Liu, On shrinking and boundedly complete Schauder frames of Banach spaces, J. Math. Anal. Appl. 365 (2010), 385-398.

[22] R. LIU AND B. ZHENG, A characterization of Schauder frames which are near-Schauder bases, J. Fourier Anal. Appl. 16, 5 (2010), 791-803.

[23] G. PISIER, Similarity problens abd completely bounded maps, Lecture Notes in Mathematics, Springer, 1995. 